\title{
Satisfying Requirements for Electronic Commerce
}

\author{
Jim Cunningham, Shamimabi Paurobally, Athanassios Diacakis, \\ Lorenz Lorenzen, Gabriel Gross, Stephen McConnell \\ Department of Computing, Imperial College, London, SW7 2BZ, UK \\ $\{r j c$, sp1, ad1\}@doc.ic.ac.uk
}

\begin{abstract}
We first present the findings of 1996 research on on-line commercial activities showing a lack of technological support for such electronic commerce. In fact, there were few signs of trading and negotiation was virtually nonexistent on-line. We also give a case study of an information retrieval system that has the potential to become an on-line brokerage service, then identify and expand the key requirements for electronic commerce. We give an overview of the $\mathrm{OSM}^{1}$ architecture for an electronic market-place and we show how its framework may be used. The paper closes with a bibliography.
\end{abstract}

Keywords: Electronic Commerce, On-line services, Brokering, OSM.

\section{Introduction}

Technology can be used in new ways to offer original and innovative services which would not have been possible otherwise. Organisations can thus gain competitive advantage. In the last few years information and communication technologies (such as Internet and the World Wide Web) have given birth to Electronic Commerce.

Electronic commerce is defined as performing commercial transactions on-line. Although still in its infancy, Electronic Commerce offers several advantages over traditional ways of doing business. Organisations perceive Electronic Commerce as a means of reducing costs and offering better services that are accessible to a wide range of market participants. However a framework for electronic commerce has to meet a number of requirements.

For participants in a market to be able to trade with each other on-line, there has to be a common framework to facilitate communication and co-ordination. This infrastructure has to meet a number of requirements.

The OSM Consortium performed a Broad Spectrum Study to understand how electronic markets work and to get a broad view of what has been happening in electronic commerce. This study was completed in early 1997 but the observations appear to be still valid. The main findings were disappointing sales patterns due to lack of interac-

\footnotetext{
${ }^{1}$ OSM is a project to create an open service model for global information brokerage and distribution under the European Commission ACTS programme, (http://www. osm. net/).
} 
tivity, advertising space being hard to sell and proprietary technology being abandoned in favour of open standards. The conclusions suggest that technology has been unsatisfactory and that there is a need for a complete infrastructure for electronic commerce, [4].

Our study found a very limited number of vending patterns in today's electronic markets. The commercialisation pattern is still nothing more than a copy of what noncommercial people think real life commerce is. Usually this is a Web page which is more or less the shop window, along with some form of catalogue and perhaps even a shopping basket. However, the Internet is an interactive medium and this is where its potential is hidden. The study did not find any sophisticated interactive process which demonstrated the new potential of the Internet and realised benefits for electronic commerce, for example, in the way hypertext enriches information access.

\section{Electronic Commerce Today}

The findings of the Broad Spectrum Study, in more detail, were:

\section{The importance of payment systems}

Security issues relating to payment are perceived as a determining factor for the proliferation of electronic commerce. Thus the main research efforts appear to be concentrated on payment systems. To be able to show up front that a secure technology for payment is being used is a sign of reliability. Yet, behaviour studies do not show this element as a determining factor for first time purchase: the potential buyer usually goes all the way through his 'shopping process' and then decides whether the payment method offered by the vendor is acceptable or not.

About $60 \%$ of the sites studied used the idea of a Virtual Caddy (Shopping Basket) for the shopping process and just under $90 \%$ accepted credit cards.

Although roughly $50 \%$ of the selected commercial Web sites used encryption technology, it is commonly accepted that more than $50 \%$ of Web-based transactions happened in insecure environments, i.e. the buyers don't know or don't care.

\section{Advertisements only at popular sites}

About $60 \%$ of the sites studied used advertising. This takes place either by advertising on other sites (referring), or by advertising products or services on one's own site. Webmasters often use the opportunity to promote specific parts of their sites.

It is common knowledge that referral links (advertisements which will take the user to a different site) are hard to sell. Only the major sites, in terms of traffic, are able to attract advertisements. 1996 studies showed that two-thirds of the advertising spending, went to the 10 largest sites. This indicates that advertising is not seen by the market to offer any real value except for a few exceptionally active sites. Even in those cases there is the problem that users don't 'click' 2 on adverts very often. In 'Wired', a reference magazine on Web life, 'Never' is defined as 'the last time you clicked on a banner ad'. This has led to a change in the pricing of Web adverts. Advertisers are

\footnotetext{
2 By 'click' we mean the user action of indicating intention to a part of the visual image, usually achieved by depressing (clicking) the button of an electronic pointing device (mouse).
} 
not willing to pay a price per person that has seen the advert. Instead they will only pay a certain price per person that has clicked on the advertisement and hence referred to the advertiser's site.

As most home users are reluctant or even not willing to pay for some online services, advertising may increase in some areas. With new technology advertisers will be able to target the right portion of the market, for example, by advertising on a small site with very few hits, but read by the right kind of people. Business users are usually quite willing to pay for useful information, so advertising may not be the only way to fund certain sites.

\section{Legal Framework}

The Internet is changing the way many organisations and individuals function. However, claims that a completely new legal framework is required to cover the "electronic world' may be excessive. It is true that the current legal system lacks certain features, but principles that apply to the Internet apply to any other areas. So widespread concerns with pornography and with copyright has elicited adaptation of pre-existing laws.

On the other hand, there are aspects that require new law. For example, if digital signatures are adopted and are deemed to be secure, laws need to be drafted to make them acceptable in a court of law.

Other matters can be raised too, such as security (anonymity and privacy mainly), trust and loss of government control. The issues involved here need clarification. Can the government be considered to be a trusted third party and do users think so too?

\section{Brokering}

The Internet enables customers to locate and purchase products and services, with less dependence on the location relative to the vendor. The main difficulty is locating the relevant products or services. This problem could be solved by special intermediary services, brokers to match vendors with consumers.

Brokering services are still rare today in electronic commerce. This is due to the lack of adopted standards. The very few services that exist appear to be business-tobusiness, not very interactive and to have crude interfaces. Although the need for brokering does exist, research concentrates on security and payment systems. Yet customers would gain access to a larger market with more competitive prices. Vendors may not be so keen, because competition reduces profit margins, but in the long term, brokering should induce access to a global market as well as providing a market more responsive to changing customer needs.

\section{Case Study of a Business Information Service}

We present here a case study to examine the requirements and constraints of brokerage-based business processes.

In this case study an industrial association provides information retrieval services to its customers. The service provider, "wf - Gesellschaft zur Förderung der Schweizerischen Wirtschaft"3 can be accessed by registered association members who pay a

\footnotetext{
${ }^{3}$ Association for the promotion of Swiss business.
} 
yearly contribution. $w f$ is a private institute that supports its association members with information about business, economics, and politics. It is located at four sites within Switzerland: Zurich, Geneva, Lugano, and Bern and has approximately 60 employees. The study was mainly performed through interviews with representatives of the wf organisation.

\subsection{Services Offered by wf}

The aim of $w f$ is to provide its external customers with on-line access and distribution of stored business information as a commercial service. As such, $w f$ is an ideal candidate to be transformed into a commercial on-line brokerage service. Its customers are able to:

- Dynamically access a local database to perform queries.

The most significant service performed by $w f$ is the provision of a document retrieval service to external persons and organisations. First, suitable combinations of thesaurus entries (e.g. "Politics", "Switzerland", and "Agriculture") is selected. This can be performed by either the customer or by $w f$ on a customer's request. Then, $w f$ retrieves a result list from the database server that contains document references and index information. Finally, $w f$ retrieves the actual image or text document from the archive server.

- Pay for the information obtained; $w f$ charges its customers on a yearly basis.

- Use this service to perform searches across multiple vendors of information. $w f$ can be thought of as a broker. It acts as an intermediary between external sources of information and its customers. However no negotiations take place.

\subsection{Actors}

The main actors in the document acquisition and retrieval process are:

- External Users

Regarding a specific branch of the $w f$ organisation, external users are either member companies or remote branches of $w f$. They perform queries either directly with a dedicated client (HYPARCHIV) or indirectly through an internal rechercheur.

- Information providers

Journalists or external staff members of $w f$ may provide content information, i.e. newspaper articles or dossiers.

- Document acquisition staff.

Newspaper articles are scanned into a temporary store (the lector's input folder). Lectors having a broad common knowledge perform the next step of document classification. The lectors aim to provide a semantically consistent document database. However, they are restricted by the terminology and lexical structure of the underlying thesaurus database. Finally, a supervisor stores the documents in the document database. The supervisor may revise or correct previous classifications. 
- Rechercheurs.

The rechercheur searches for documents using queries. He uses the thesaurus database to formulate a proper query. After having selected suitable combinations of terms (such as "Politics", "Switzerland" and "Agriculture"), the rechercheur accesses the document database. He may refine his query or access the documents denoted by the most recent result.

\section{- Administration}

The administrator maintains the Thesaurus and Document databases in order to reorganise storage mechanisms.

\subsection{The Value Chain of Document Processing}

The current document processing approach can be modelled as a value chain:

- Publishers provide information through newspapers and specialised magazines. The paper-based format is passed on to $w f$.

- $w f$ provides acquisition, classification, and retrieval services.

- External users may either retrieve their documents individually or they can send an order to $w f$ s rechercheurs for retrieval.

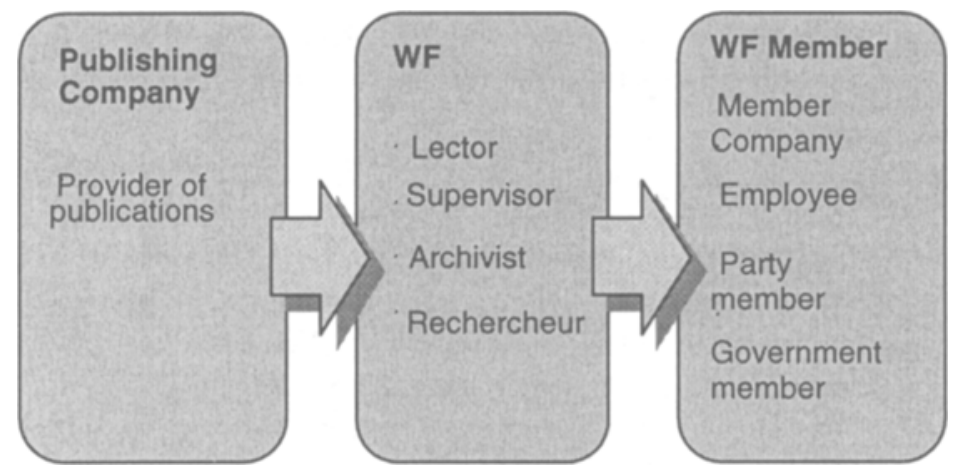

Fig. 1. Value Chain of wf

\section{The impact of Information Technology}

$w f$ relies on Information Technology and uses Electronic Document Management (EDM) systems. EDM is the process by which a paper-based image is converted into an electronic format and processed by computers.

\subsection{Technical components of wf}

$w f$ consists of the following components:

- HYPARCHIV - The Document Management System

The real-world organisation of archives, files, folders, stapled and elementary documents can be mapped correspondingly into HYPARCHIV, the electronic archive. HYPARCHIV thus handles up to several hundred gigabytes of direct access storage. 
A query processing function allows users to interactively refine queries. Users can also visually select output from result lists and combine it logically with other result lists. HYPARCHIV can be connected to other applications such as database servers, document processors, or accounting applications.

- The technical infrastructure

The $w f$ network consists of 4 sites including one in Zurich. The Zurich network has a central file server managing a document cache disk, an archive server, database servers and fax servers amongst others.

- The Thesaurus

The $w f$ thesaurus database is a uniform, neutral and multi-lingual document classification. It provides a user interface that allows a simplified navigation through its hierarchical structure. The supervisor is able to augment the thesaurus structure by registering new concepts within the thesaurus hierarchy. The classification of dossiers and book publications is carried out by entering descriptor information into fields.

\subsection{Requirements for an Electronic Information Service}

- Support for decentralisation

In the case of $w f$, all publishing activities are done in-house. The documents are collected from newspapers and magazines and then processed within $w f$. In a decentralised scenario, it would be possible to subcontract an activity like document scanning.

- Payment systems

wf at the moment charges on an annual subscription basis. No additional fees are charged for individual retrieval activities. This method of payment is acceptable when service utilisation is stable and when the growth rate of the overall service utilisation is moderate. However when customers are charged at the rate they access the site, the overall costs are distributed in a more realistic way. Yet, according to $w f$, charging on a per-session basis would cause higher transaction costs for accounting procedures because their computer systems cannot at the moment log user sessions. Hence the subscriber model is at present preferred at $w f$.

- Security

The HYPARCHIV software system supports the authorisation and authentication of internal and external users. Since external users only access the database/archive server through ISDN telephone lines, the possibility of fraud or masquerade is reduced to a tolerable level. Alternatively, a data encryption system can be used in order to prevent unauthorised parties from interception.

Since payment is effected on a subscriber basis, the on-line activities do not involve any financial transfer. Therefore, no incentive or reason for repudiation is present in this case study.

- Fast retrieval

The user may obtain the document by retrieving search results individually and ordering them to be delivered through fax or mail. The HYPARCHIV software provides an average access time between one and 10 seconds with a variation of up to 30 seconds delay per storage medium. The overall access time depends on the location of the 
document, the complexity of the query, the image decompression effort and the data transmission rate through the telephone lines.

- Standardisation

A specific user tool - the wfBASE client - is required to access the archive server because the overall services provided are not standardised. However, the thesaurus structure is standardised and navigation through its hierarchical structure is possible.

- Multilingual support

HYPARCHIV is multilingual since the user interface language can be changed at runtime. Languages such as English, German, French, Italian, Spanish, Portuguese, Dutch, Serbo-Croatian, Swedish and many more are supported.

\subsection{Conclusion from the Case Study}

This study illustrates the information activities offered by an industrial association through the usage of EDM systems. Compared to search engines such as Lycos ${ }^{4}$, DEC's AltaVista ${ }^{5}$ or Yahoo $^{6}$ which have on-line indexes of 20-100 million documents and which receive approximately 100,000 queries per day, the $w f$ database captures only a fraction of these amounts. Yet, the value of the information stored is much higher, since the classification by human lectors assures a higher selectivity and quality. $w f$ provides added value to existing information by selecting, classifying and indexing the documents stored. Business customers are willing to pay a high charge for comfort and for human expertise in both the processes of document acquisition and retrieval. So in an Internet-oriented setting, the integration of human resources may be an important factor within the preparation and provision of services.

Payment is not charged proportionally to the use of the service but rather on an annual subscriber basis. This method is feasible in the setting of a stable service utilisation, but has in high costs. Potential customers may be discouraged from performing retrieval tasks.

The per-year subscriber payment model reduces the risks of fraud and hence simplifies the procedures for authorisation and authentication. However this reduction in risks benefits only $w f$ customers. For example, if a third party connects to the $w f$ service using the account of a $w f$ member, the member will not be charged more, but $w f$ will incur the extra cost.

In relation to the investigation and analysis carried out, we can extrapolate the example of $w f$ to that of a commercial on-line brokerage service. The different functions involved in information retrieval such as acquisition, registration, archiving, retrieval and distribution may be decentralised and accessed separately from various locations. Then $w f$ would act as a broker and combine the back end services to offer an integrated service to a business customer.

\footnotetext{
${ }^{4}$ http://www.lycos.com/

${ }^{5}$ http://altavista.digital.com/

${ }^{6}$ http://www.yahoo.com/
} 


\section{Requirements for Electronic Commerce}

Although studies suggest that most requirements apply to any sector, there are some sector specific requirements. For example:

- Banking \& Insurance: Different laws on the protection of security of funds apply in different countries.

- Insurance: Complex mutual transactions which are negotiated and performed online need technical support (e.g. in the areas of reinsurance and co-assurance).

- Publishing: External parties with neutral judgements (e.g. rating services) are required in a decentralised environment to evaluate and recommend service providers for the quality of their services. Rating services may be useful in other areas too.

We have ranked the requirements that apply to most sectors. The rankings are based on the analysis of data collected from studies, including the Case Study presented in the previous section. The rankings are indicative and not absolute.

In the first list the features are ranked by their current level of support. Those that are best supported come first. In the second list, they are ranked according to the preferred support level. Finally, in the third list the ranking is by the effort required to reach the preferred level of support. Those features that are not currently well supported but are required are given first. They are followed by those features that are required less but might be fairly well supported.

\section{Currem level of suppon (perceived)}

1. On-line distribution

2. Perfornance \& Reliability

3. User Friendliness

4. Interoperability \& Heterogeneity

5. Payment

6. Privacy

7. Human Resource Integration

8. Catalogues

9. Logs \& Documentation

10. Auditing

11. Service Brokers

12. Cerlification

13. Negotiation

\section{Preferred level of support}

1. Certification

2. Payment

3. Performance \& Reliability

4. User Friendliness

5. On-line distribution

6. Human Resource Integration

7. Interoperability \& Heterogeneity

8. Auditing

9. Privncy

10. Calalogues

11. Logs \& Documentation

12. Negotiation

13. Service Brokers

\section{Iffort Required (adjusted)}

I. Certification

2. Auditing

3. Negotiation

4. Payment

5. Human Resource Integration

6. Service Brokers

7. User Friendliness

8. Catalogues

9. Interopernbility \& Heterogeneity

10. Logs \& Documentation

11. Privacy

12. Performance \& Reliability

13. On-line distribution

We discuss the some of the features that require most effort below:

\section{Security: Certification}

Currently, the most inhibiting factor for electronic commerce is the lack of security. Security implies several things: privacy, authentication and non-repudiation. To achieve all of the above an infrastructure for security is required. The starting point for this infrastructure would be a way to identify individuals when needed. This could 
make them accountable for their actions and provide authentication and nonrepudiation. Privacy is relatively easier to achieve.

It is very likely that public key cryptography is going to be the basis of security systems. The main problem of public key cryptography is the distribution of public keys. One must have a way of knowing that a certain public key belongs to a specific individual. A Certification Agency acting as a trusted third party can certify the ownership of public keys.

An electronic commerce framework would necessarily need to support certification for the purposes described above.

\section{Auditing Support}

An electronic commerce architecture must have some support for audits in order to ensure that contracts are executed as set out in their terms and conditions. If necessary one should be able to check that an exchange was fair and that the legal formalities were taken care of. One way to do this would be to use notary services. Such services are currently non-existent in the on-line world.

\section{Negotiation}

Negotiations between humans are usually slow and may be tedious. This is especially true in complex situations. Currently there is no support for on-line negotiations. The automation or partial automation of negotiations is required. The protocols and methods used need to be standardised.

\section{Payment}

One of the greatest problems of electronic commerce is said to be the lack of secure payment systems. This is not absolutely true, as the real problem lies in the support of the payment systems and not the existence of payment systems as such. In other words, there is a lack of a framework which supports multiple payment systems. Within such a framework, consumers can negotiate with the vendor and agree on the preferred payment mechanism. Currently, there are various payment mechanisms, the most popular of which is payment with credit cards. Others have not yet reached their critical mass and as a result vendors are reluctant to use one system or another in case it becomes obsolete with the invention of something different.

\section{Human Resources Integration}

Ideally, customers want to pay only for added-value services. In some cases it is possible to add value without human intervention. Nevertheless, the 'intelligence' of computers remains limited. It is necessary to integrate human resources or to make use of human expertise. The $w f$ case study shows that business customers would be willing to pay for human expertise. It might even be possible to extend this to the mass consumer market. In any case, when computers are commonplace, the addedvalue will come mainly from humans. A framework for electronic commerce would have to be able to integrate different types of resources such as human and computer resources.

\section{Brokers}

Some types of on-line brokering already exist, for example Internet search engines. In the area of electronic commerce, brokers may be somewhat ahead of their time. Due 
to problems with features such as payment technologies, the number of vending patterns on the Internet is small and hence the need for brokering has yet to be felt. On the other hand, this is not necessarily true for all industries. Apparently, the need for brokering services has already been felt in the transport and logistics sector, mainly due to its decentralisation. We expect that vendors and consumers will realise the importance of brokering once payment and security issues have been resolved.

\section{Catalogues}

Electronic catalogues can provide complete and up-to-date information, greater choices, wider user access and on-line enrolment. Currently, electronic catalogues do exist, but they lack standardisation. As a result, each catalogue may require different hardware and software and have a different interface. Additionally they may not provide all the necessary functions and it may not be possible to integrate them with other catalogues. Also, the product and service descriptions in the catalogues might be incompatible too. As catalogues would be the main way of displaying the profiles of different services, it is imperative that some standardisation takes place.

\section{Interoperability}

With the diverse range of existing and future systems, it is crucial that any electronic commerce framework is not platform specific. Adoption of standards such as those of the Object Management Group will ensure interoperability across a wide range of computer systems. An electronic commerce framework would be based on the idea of a global network and to achieve that, interoperability is a prerequisite.

\section{Intent of the Open Service Model}

The open service model aims to provide a coherent and inter-operable framework for electronic commerce.

- Software developers and technology providers need a reference architecture to use for the development of their products. The architecture must be standardised enough to allow them to implement parts of an overall electronic market system and at the same time flexible enough to allow the market infrastructure to evolve.

- Consumers need a wider choice of products and services at competitive prices and a market that will adapt quickly to their needs. They should be able to make purchases of standard and non-standard services, as well as compound services to suit their individual requirements.

- Providers need the means to access a large, global market at very low cost, 24 hours a day, every day, to offer services efficiently and effectively and shorten drastically their product cycle times. Providers must be able to publish their services without the need to formally standardise them.

\subsection{Business Transactions}

Business transactions are broken down into two phases in the OSM framework; the service construction phase and the service execution phase. These can be summarised as follows: 
- Service Construction

Information Collection. Initially, information about the relevant products or services is exchanged between the parties involved. This can include advertising, product brochures, electronic catalogues and others.

Negotiation \& Agreement. Once the required information has been collected, parties start negotiating about the terms and conditions of the transaction. These terms and conditions form a contract.

Engagement. The final step of this phase is the engagement, when all parties accept the contract and agree to be bound by its terms.

- Service Execution

Arrangements. Firstly, if not already agreed upon, common policies such as method of payment, place of delivery and security policy are settled.

Execution. In this step the commercial services are provided, within the context of a higher level policy such as that of national law or fair exchange. Usually, the vendor will supply some products or services and the customer will pay for them.

Termination. The execution of the contract is validated according to the policies concerning delivery assurance or fitness for purpose. The contract is then terminated.

In most cases, contracts are implied but they are not set out explicitly. When one buys a newspaper from the newsagent, there is an implied contract: i.e. the customer can have the newspaper if he pays they newsagent a certain amount of money. Current electronic commerce operations, such as those on the World Wide Web, primarily support the information collection step. There is little or no support for the other steps. Under the OSM framework, sophisticated mediators (brokers) can make the exchange of information between consumers and providers of services quicker, cheaper, more secure and effective during both the construction and execution phases.

\subsection{Electronic Brokering}

OSM has specified an architecture to support brokering in electronic commerce. Electronic brokers help to provide value-added services to deal quickly and effectively with the vast amount of information available about products and services which would otherwise be difficult, if not impossible, to search. These brokering agents help to overcome the limitations of direct negotiations between customers and suppliers through matchmaking, negotiating and monitoring transactions.

In the OSM architecture, brokers will help in each step of both the service construction and execution phases. For example:

In the information collection step:

- A broker may provide value-added services by maintaining databases about products and services. This information can then be selectively directed to the customers. So electronic brokering can cut down on expensive searches of the huge amount of information available on the Internet.

- Information about a product which is gathered from sources other that the product provider may be offered to consumers. This could be information from an inde- 
pendent consumer association, from a previous buyer of the product or from special rating services.

In the contract agreement step:

- Brokers may assist with negotiations. They can maintain the anonymity of the participants during negotiations by acting as intermediaries. Brokers thus enable competitors to negotiate when they may not otherwise do so.

In the contract execution step:

- Brokers may act as trusted third parties who ensure that transactions are carried out as agreed and that the consumers and providers complete their part of the deal.

Services which may be offered by brokers are:

- Registering subscribers, service offers and requests.

- Authenticating subscribers.

- Matching the service offers and requests.

- Helping in the negotiation of contracts and thus ensuring a good deal between the consumer and the provider.

- Controlling the execution of transactions. They control the settlement and execution of the contract and as a result they may be paid a percentage of each transaction.

\subsection{Scenario of wf as a broker}

The decentralisation of functions which are currently centralised such as information acquisition, registration, archiving, retrieval and distribution may lead to a role of $w f$ as a broker of on-line services. A customer may ask for an offer that combines a retrieval assistant, several Internet-based information sources and a distribution service that is integrated from a distinct provider. A first scenario may look like this:

1. Oliver forms the following request "please provide me with information on <research projects which focus on the on-line brokerage and delivery of services $>$ ".

2. $w f$ proposes: "I have found 12 documents that match your specification with three keywords. The overall number of pages is 45 . The overall charge will be $12 \mathrm{SFr}$. I can offer it to you either by email or by surface mail. In the second case, an additional charge of 6 SFr will be made. Courier shipment will cost additional 20 SFr. Additional printed copies will cost 3 SFr each. I can further offer you payment by Credit Card (AMEX, Visa, MC) or by Ecash".

3. Oliver orders the second offer with standard delivery. He chooses Ecash. He selects 5 copies. He pays 27 SFr.

4. $w f$ transfers the data to a print service in Finland. From there, it is delivered to Oliver the day after.

A second scenario may even deal with information brokers as a subject of service brokers: 
1. Oliver forms the following request "please provide me with a reference to someone who can give me information on <research projects that focus on the on-line brokerage and delivery of services $>$ ".

2. The $w f$ broker responds with the following offers: "I have found 5 information brokers which can provide more than 5 documents at less than 20 SFr".

- I can offer you this list of brokers for $0.20 \mathrm{SFr}$, or

- to inspect the current results for $0.10 \mathrm{SFr}$, or

- to compile a dossier for 40 SFr.

- If you prefer to involve an assistant, I will charge an additional 2 SFr per minute".

\section{Components of the Reference Architecture}

The Reference Architecture constitutes a formal response of the OSM Consortium to the Object Management Group's Electronic Commerce Domain Task Force RFI-2. 96-11-03 entitled Enabling Technologies and Services for Electronic Commerce. The Open Service Model will standardise the following features of on-line trading: payment and security facilities, transactions and persistent asynchronous communications and catalogues \& services. The architecture will be based on CORBA 2.0 distributed computing infrastructure. Implementation of the architecture will provide tools for client interaction with service providers, service access, catalogue maintenance, negotiation and monitoring of service delivery, intermediate brokers supporting standard and non-standard services, intelligent service matching and dynamic service construction.

The OSM architecture is composed of three principal layers:

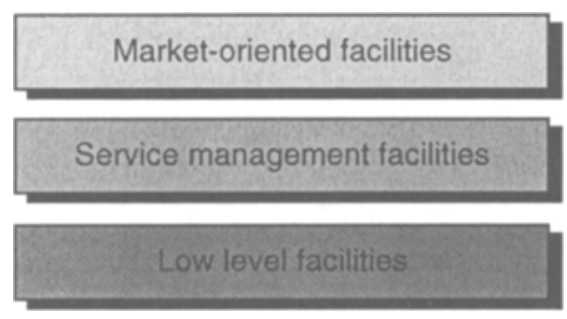

Catalogue, Agency and brokerage facilities

Object browser, session, contract and service management facilities

Negotiation, selection, certificate and payment facilities

Fig. 2. Three Principal Layers in the OSM Architecture

The architecture and supporting tools are currently being implemented. Public infrastructure pilot trials will validate the architecture and its implementation.

\subsection{The Facilities supporting the proposed Standard}

The OSM architecture is centred around the CORBA 2.0 specification. This implies that, under the standard, communication between participants proceeds via the IIOP 
protocol. Implementation of the OSM architecture uses the Business Objects Facility (BOF) interoperability ${ }^{7}$ specification from the OMG as framework. OSM facilities are implemented over the BOF framework implementation to facilitate on-line trading.

Session facilities support asynchronous event communications by opening a channel between the participants providing transactional integrity and channel security. Providers of services advertise their products on the relevant information channels and authorised consumers subscribe to channels of interest.

The object browser provide the framework for presentation and management of components such as services, contracts, certificates. This offers portability and interoperability of products and services across desktop environments.

It is intended that participants in electronic commerce search, filter, gather information, navigate and exchange contracts and services. Catalogues enable information browsing and inspection through their interface. Collections of items from different suppliers can also be constructed into a catalogue, so enabling brokerage. An agency facility establishes a formal query point and public query interface about a provider in an electronic marketplace.

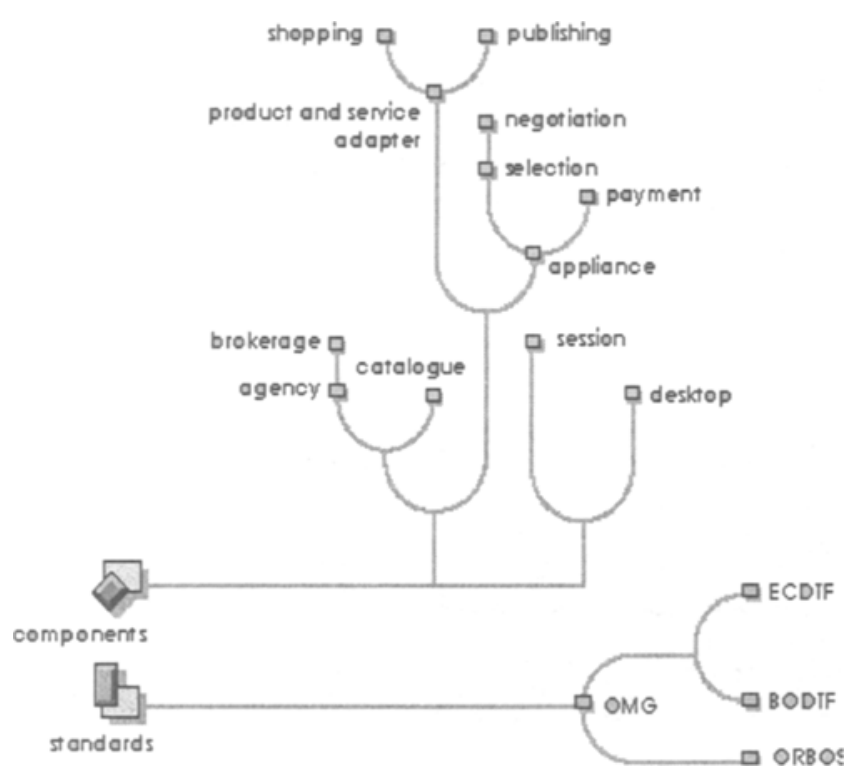

Fig. 3. Tree of the OSM facilities

The brokerage facility introduces the concepts of discovering and advertising resources. This facility allows users to be more focused in dealing with information about commercial services and provides anonymity and privacy of the transaction processing.

\footnotetext{
${ }^{7}$ Business Objects Architecture Interoperability Specification, OMG Document. ftp://www.omg.org/pub/docs/bom/97-09-01.doc
} 
The selection facility supports selection and configuration of supporting appliances and policies. The negotiation facility allows parties to communicate and mutually agree on a transaction.

The payment facility enables interactions between a buyer and a seller and any necessary third parties for the successful electronic exchange of goods or services. The value exchanged is governed by regulations and business requirements which are expressed in a "payment protocol" associated with each payment type.

\section{Two Scenarios of on-line commerce under OSM}

Here we highlight some features of the OSM architecture by presenting two imaginary scenarios. The underlying principles should be supported by an electronic commerce infrastructure. In the first scenario, a user called Donald is interested in buying a pizza on-line. Donald should be able to carry out his purchase quickly and easily. In contrast, the second scenario shows a sample of the negotiation between an author and a publishing agent in electronic publishing.

\subsection{Donald buys a Pizza on-line}

\section{Opening the Browser}

We assume that Donald has already acquired the software that enables him to trade in electronic commerce. Donald launches his favourite object browser which represents the user interface to enter the online trading market. This may be done by clicking on the icon representing the electronic commerce software on his desktop. A connection window appears prompting Donald for his login and password. After he has entered his password and asked for connection, the underlying OSM facilities perform authentication checks. When Donald has successfully logged on, the desktop is usertransparently configured. This involves launching supporting appliances such as payment, selection and negotiation facilities and loading Donald's particular preferences, for example links to his favourite sites, his saved and incomplete transactions or his preferred layouts.

\section{Browsing the Services}

The main browser window now shows a top level catalogue with the services available on the market. Donald can either enter a IIOP URL for a site that he wants to visit or recursively browse through the offered options. He chooses to adopt the second method in this case. He selects the food section and more specifically take-away restaurants of Italian kind. Furthermore he enters the keyword 'Pizza' to denote his particular interest. Working in a way similar to web search engines, the browser returns a list of restaurants and their representative icons offering pizzas purchase online. Mario's Pizza, Pizza Hut, Pizza Paradise, Mama Cabella's Pizza Emporium are the restaurants that form part of the given list. Donald is curious about the restaurant called Mario's pizza and selecting its icon, he clicks on the inspection mode option offered at the bottom of the browser. A window pop-ups giving details of Mario's pizza restaurant such as the name, location. 


\section{Inspection and Presentation}

Donald now chooses to browse through the services offered by Pizza Hut. He double clicks on Pizza Hut and a catalogue of the pizzas from Pizza Hut is displayed. Donald can choose to inspect the general statistics of a pizza by selecting it and choosing the inspection view. What he sees is the composition of a pizza, possible additional toppings and the prices in a standard format.

Donald also can see a customised view of the pizza by selecting a pizza and the presentation mode option at the bottom of his browser. The browser displays a picture of the selected pizza with the price in a presentation window. Donald then drags icons representing toppings onto either the inspection or presentation views to compose his own pizza.

\section{Buying and Paying}

When Donald is satisfied with the pizza toppings, size and the price, he clicks on the buy button to show he is interested in paying right away. Or if Donald wants to browse, selecting and buying other services before paying, he launches a payment cart offered on the menu. He then selects the icon for the pizza that he has composed, drags and drops it onto his payment cart. From then on any service that he chooses to buy, he can drag and drop its icon onto his payment cart. Finally, when Donald is ready to pay for his purchase/s, (with or without having constructed a payment cart order entry), the composition, delivery time and price of the pizza he chose are displayed. Here the underlying payment facility of OSM is called. Donald agrees with the purchase, he clicks on the pay button and is prompted to enter his user payment details. If validation is successful, delivery of the pizza signals the completion of the transaction. Donald's bank will get a request together with Donald's personal payment details to credit the price the pizza cost from Donald's account to Pizza-Hut.

\subsection{Negotiating in Electronic Publishing}

In this scenario, transaction costs are assumed to be negligible and hence the accessibility of services is not inhibited by them. The resulting decentralisation changes the roles of the actors.

\section{Actors in this scenario}

A single person may offer a variety of services to consumers, acting as a proof reader, a lector or a reviewer and may even have several roles at the same time.

- The author can directly employ the services mentioned above. Publishing services can be provided directly to the readers and the author may also employ supporting services such as formatting, advertising, marketing, distribution or accounting.

- A publication agent provides the author with a simplified set of services as opposed to the multitude of services offered on the network. This agent can propose a combination of services required to publish a document. The publishing agent can offer different levels of quality for the services he provides. The author can reject this offer or combine it with other services. A contract may be agreed upon after negotiation between the broker and the author. The author may make only one payment or may pay in stages. Publishing agents may also undergo reviewing and rating. 
Author: [Establishes a connection with the broker and exchanges certified keys] Publish my document (attached).

Broker: [The broker consults the author's profile with his usual requirements]

I will do the following for you (it will cost $220 \mathrm{ECU}$ ):

Engage an advertising agency.

Carry out a catalogue service registration.

Engage a proof reader.

Ask up to 10 reputed persons for a statement.

Author: Your proposal is acceptable if you additionally provide the following:

The document must be accessible through a $2 \mathrm{Mbit}$ channel (or faster).

I want to select the advertising agency.

I want to receive at least 5 ECU per issue sold.

I want to pay by Mondex and be paid in ecash.

Broker: Select one of the following (sorted by ratings provided by Good Service Ltd.):

Sterenberg \& Co, Ltd.

City Media Advertising, Inc.

Marquand, Gottesman \& Duhaut.

Katumba \& Katumba.

[More options]

$5 \mathrm{ECU}$ per issue is not wise. I suggest $2 \mathrm{ECU}$, modified proposal attached.

Author: $\quad \mathrm{OK}$, drop 5 for 2 .

I'll use Katumba \& Katumba.

Broker: $\quad$ For payment modalities, I recommend the model conditions of the European Publishers Association (see attachment).

Author: $\quad$ OK, proposal is acceptable. Make me an offer.

Broker: $399 \mathrm{ECU}$, (whole proposal attached).

Author: Too expensive. I agree for $299 \mathrm{ECU}$, proposal attached.

Broker: $\quad$ No, 349 ECU, proposal attached.

Can offer 20 Air-Miles if you agree to review the services you receive.

Author: Accepted. Will take Air-Miles offer.

Broker: Attached is the notarised contract.

[after 2 days]

Broker:

Proof-reading completed. Please review changes (attached).

Author: Please incorporate changes (instructions attached).

[after 3 days]

Author: Report current status.

Broker: $\quad$ Proof reading (completed).

Advertising agency services (completed).

Catalogue registration (pending).

Statements received: $7 / 10$ (pending).

[after 4 days] 
Broker: All services performed (see consolidated activity record). [after 1 week]

Broker: $\quad$ Please review the following services you received, forms attached.

Author: Review forms attached.

\section{Conclusion}

Studies show that the full potential of the Internet as an interactive medium for conducting online transactions is not being exploited. Electronic commerce is restricted to information collection and perhaps even a shopping basket. There is no support for negotiation. Brokering services are still rare.

Security, implying privacy, authentication and non-repudiation, is one of the most important requirements for electronic commerce. The security of an underlying infrastructure will determine its adoption. Participants want frameworks that will preserve their own privacy and anonymity as well as guaranteeing secure payment mechanisms. Another major requirement is the need for electronic brokers. Brokers cover a range of functionalities such as support for negotiation, matchmaking, effective information search and the supervising, drawing up and execution of contracts. Such services are currently absent in the online world.

Our information retrieval case study highlighted the fact that human expertise is still needed to provide added value to information. Thus any framework for electronic commerce must provide for the integration of human resources. Business customers are willing to pay for human expertise.

An electronic commerce infrastructure needs to be inter-operable with and make full reuse of the different technologies available. In its first stages, electronic commerce may be an extension of traditional commerce. The electronic framework must be sufficiently standardised for practical use but be generic enough to support market evolution and emerging business models.

The OSM project provides a framework for conducting transactions on-line. An open architecture has been specified and is being implemented. Since this architecture is centred around the OMG's CORBA 2.0 and BOF interoperability specifications, the requirements for mobility, portability and interoperability are satisfied. Furthermore, the OSM architecture aims to satisfy the requirements for negotiations and electronic brokering. The need for an integrated security system will make use of the OMG security specification.

Thus, electronic commerce frameworks such as OSM will allow buyers to conduct transactions as simple as buying a pizza on-line. One should be able to carry the purchase easily and quickly with a user-friendly interface. A pizza buyer need not be concerned with security, installation or validation algorithms. Likewise service providers want an infrastructure that will allow them to customise their presentation as well as be accessible to a wide range of potential customers. Yet, an electronic commerce framework must also support complex transactions involving negotiation, price fluctuations and brokering such as in auctions. OSM aims to support both the simple and the complex requirements described above. 


\section{Acknowledgements}

Material from project deliverables "D1 - Case Studies", "D2 - Business Process Studies", "D3 - Broad Spectrum Study", "D6 - Consolidated Analysis" and " OSM Reference Architecture" has been adapted for this paper. The authors constitute the main direct contributors but we wish to acknowledge help from other members of the OSM project. While prepared in good faith any opinion expressed in this paper is that of the authors and not necessarily a faithful representation of any party to the project.

\section{Bibliography}

1. ACS Systemberatung, "Electronic Document Publication and Distribution with Electronic Libraries", OSM Report, 1996.

2. OSM Consortium, WP1 Task 1.1, Deliverable 1 Case Studies.

3. OSM Consortium, WP1 Task 1.2, Deliverable 2 Business Process Studies.

4. OSM Consortium, WP1 Task 1.3, Deliverable 3 Broad Spectrum Study.

5. OSM Consortium, WP1 Task 1.6, Deliverable 6 Consolidated Analysis.

6. OSM Consortium, WP2, OSM Reference Architecture.

7. Birmingham, W. P., Durfee, E. H., Mullen, T., et al., "The distributed agent architecture of the University of Michigan Digital Library", AAAI Spring Symposium on Information Gathering from Heterogeneous, Distributed Environments, Stanford, CA, AAAI Press, 1995.

8. Chaum, D., Fiat. A., Naor, M., "Untraceable Electronic Cash" in S. Goldwasser, Hrsg., Proc. CRYPTO'88, Springer LNCS 403, Berlin Heidelberg New York, 1990.

9. Fidel, R., Bellardo, T., Rasmussen, E. M., Smith, P. J., Challenges in Indexing Electronic Text and Images, ISBN 0-938734-76-8.

10. International Standards Organisation, Filing and Retrieval (DFR) - Part 1: Abstract service definition and procedures.

11. OSM web site, http://www.osm.net/.

12. International Standards Organisation, ISO 2788: 1986 Documentation - Guidelines for the establishment and development of monolingual thesauri

13. International Standards Organisation, ISO 8459-3: 1994 Information and documentation - Bibliographic data element directory - Part 3: Information retrieval applications.

14. International Standards Organisation, ISO/DIS 23950 Information and documentation - Information retrieval (Z39.50) - Application service definition and protocol specification.

15. Janson, P., Waidner, M., "Electronic Payment over Open Networks", INFORMATIK - Zeitschrift der Schweizer Informatikorganisation, 1(3) June 1995, pp. 10-15.

16. Jones, S. et al., "Interactive thesaurus navigation with intelligence rules", Journal of the American Society for Information Science 46(1), January 1995, pp. 52-59.

17. Kelly, K., Out of control: the new biology of machine, social systems and the electronic world, Addison Wesley, 1994. 
18. Malon, T., "Modelling co-ordination in organisations and markets", 1986.

19. Malon, T., "Computers, Networks and the Corporation", Scientific American, September 1991.

20. Medvinsky, G., Neuman, B. C., "NetCash: A design for practical electronic currency on the Internet", Proc. 1st ACM Computer and Communications Security, Nov. 1993, ACM Press, New York 1993, pp. 102-106, http://www.netcheque.org/info/netcash.

21. Medvinsky, G., Neuman, B. C., "Requirements for Network Payment: The NetCheque TM Perspective", Proc. IEEE CompCon '95.

22. Miller, M.S., Drexler, K. E., "Open Agoric Systems", B.A. Huberman: The ecology of computing, Elsevier, Amsterdam New York, 1988, pp. 133-176.

23. Milstead, J.L., ASIS Thesaurus of Information Science and Librarianship, ISBN: 0-938734-80-6.

24. Mullen, T., M. P. Wellmann, Some Issues in the design of market oriented agents, University of Michigan, 1996.

25. Mullen, T., Wellman, M. P., “A simple computational market for network information services", First International Conference on Multi-agent Systems, San Francisco, CA., 1995.

26. NISO/ANSI/ISO 12083, Electronic Manuscript Preparation and Markup, ISBN 1880124-20-3.

27. Sairamesh, J., Nikolaus, C., Ferguson, D. F., Yemini, Y., "Economic Framework for Pricing and Charging in Digital Libraries", D-Lib Magazine, February 1996, ISSN 1082-9873.

28. Sirbu, M., Tygar, J. D., "An Internet Commerce System Optimized for Network Delivered Services"., Proc. IEEE CompCon, March 1995.

29. Sirbu, M., Tygar, J. D., "NetBill: An Internet Commerce System Optimized for Network Delivered Services", IEEE Personal Communications, 2(4), 1995 , pp. 34-39.

30. Steiner, J. G., Neuman, C.B., Schiller, J. I., "Kerberos: An Authentication Service for Open Network Systems", Proc. of the Usenix Winter Conference, Berkeley, CA, USA, Technical Report MIT 1988, pp. 191-202.

31. Varian, H. R., "Economic mechanism design for computerized agents", Proc. First USENIX Workshop of Electronic Commerce, USENIX Assoc., Berkeley, CA, USA, 1995, pp. 13-21.

32. Waidner, M.: "Development of a Secure Electronic Marketplace for Europe", EU ACTS Project SEMPER, Document No. 431ZR056, 19. February 1996, http://www.zurich.ibm/. 